\title{
Super-resolution imaging of nanostructures by solving the phase problem with over-sampling of diffraction patterns
}

\author{
J.M. Zuo, ${ }^{*}$ I. Vartanyants, ${ }^{* *}$ M. Gao, ${ }^{*}$ L.A. Nagahara*** and R. Zhang*** \\ *Department of Materials Science and Engineering and Materials Research Laboratory, \\ University of Illinois at Urbana-Champaign, IL 61801 \\ ** Department of Physics and Materials Research Laboratory, University of Illinois at Urbana- \\ Champaign, IL 61801 \\ ***Physical Sciences Research Laboratories, Motorola Labs, 7700 South River Parkway, Tempe \\ AZ 85284
}

Atomic resolution imaging can reveal critical structural information underlying important materials properties or interactions fundamental to our understanding of natural processes. Developments in electron microscopy have made imaging of atoms in periodic crystals at resolution close to $1 \AA$ resolution a routine in many materials laboratories. However, many nonperiodic structures, like molecules and nanostructures, lack well-defined atomic columns in a periodic crystal to be imaged in projection and/or the scattering of atoms is too weak to yield significant contrast. Imaging atoms in a molecule, thus, requires a much higher resolution and sensitivity.

Single atom imaging for structure determination is currently performed by x-ray and neutron diffraction, which averages over many identical cells and focuses beams into directions defined by the crystal reciprocal lattice. This is applicable for particles like certain macromolecules that can be synthesized in a homologous series and crystallized into a periodic crystal. For dispersed single particles, cryo-electron microscopy at $\sim 7 \AA$ for highly symmetrical virus is obtained by averaging over many low-dose images of molecules of the same configuration. Resolution is limited by low signal/noise ratio set by radiation damage and by other instrumental factors like lens aberration and sample drift. Nanostructures, such as carbon nanotubes, come with different sizes and structure. Their structure determination, thus, requires the ability to select and record high-resolution data from individual nanostructures, which up to now is lacking. As a result, many nanostructures have not been accessible by crystallography.

Here we will report a feasibility study of single molecular diffraction and atomic resolution imaging from a diffraction pattern using coherent electron nanodiffraction. From the studies of Bates [1] and others [2], we know that the phase problem is uniquely solvable in more than one dimension for localized objects. Unique phase can be retrieved by an iterative procedure from a oversampled diffraction pattern using the procedures pioneered by Gerschberg-Saxton [3] and developed by Fineup [4]. Since there is no image-forming lens involved and diffraction pattern records structure information at much higher frequency than direct imaging, this technique appears to solve many technical difficulties in conventional imaging, including resolution limit by lens aberration, sample drift, instrument instability and low contrast in electron image [5].

\section{References}

[1] R. Bates, Optik, 61, 247 (1982)

[2] For example, see J. Miao, P. Charalambous, J. Kirz, D. Sayre, Nature 15, 342 (1999).

[3] R.W. Gerchberg and W.O. Saxton, Optik 35, 237 (1972).

[4] J. Fienup, Appl. Opt., 21, 2758 (1982).

[5] Research supported by DOE DEFG02-01ER45923 and DEFG02-91ER45439 and uses the TEM facility of Center for Microanalysis of Materials at FS-MRL. 


\section{Over-sampling for a finite-sized object}

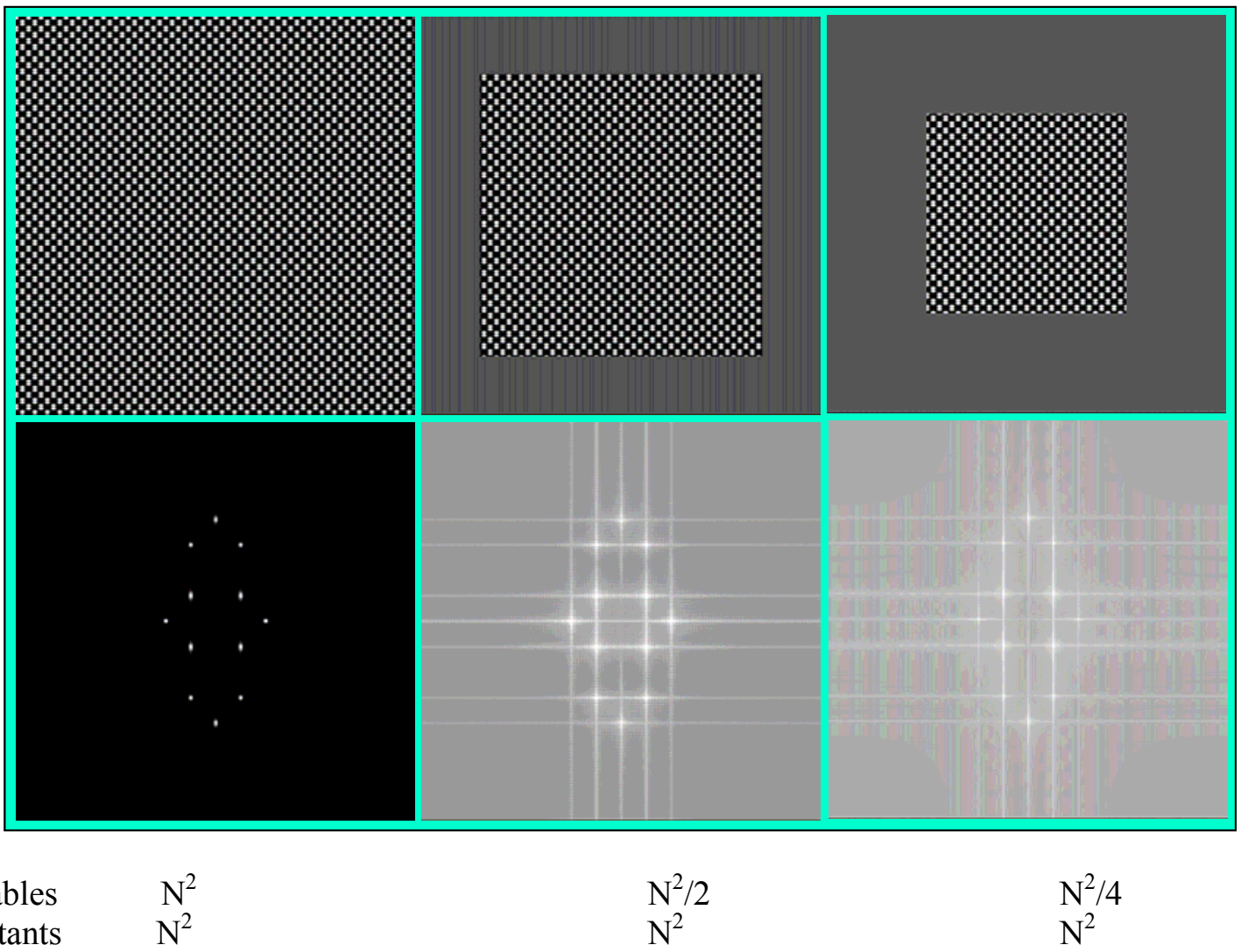

FIG. 1. An illustration of over-sampling. Top row left) a periodic image sampled by NxN pixels, middle) a finite sized image occupying half of the $\mathrm{NxN}$ pixels; right) a finite sized image occupying a quarter of the NxN pixels. Bottom row) Fourier transform of the top row. In a diffraction experiment, the amplitudes of the Fourier transform are recorded, which gives $\mathrm{N}^{2}$ constants. Fourier transformation for image reconstruction requires both amplitudes and phase, thus $2 \mathrm{~N}^{2}$ constants for a periodic image. The number of known's is less the number of constants required, this is known as the phase problem. Studies by Bates and others (see references 1-4) have shown if the image is finite and the number of variables is large than number of constants, then the phase can be retrieved iteratively without any prior knowledge. Experimentally, this requires a recording of diffraction pattern at resolution $1 /(\mathrm{L}+\mathrm{S})$, with $\mathrm{L}$ as the length of the object and $\mathrm{S}$ as the size of the supporting area. Since a Fourier relationship is assumed between diffraction pattern and image, this also requires a coherent illumination over the length of $\mathrm{L}+\mathrm{S}$ and kinematic approximation for scattering. 